\title{
When Anesthesiologists Should Stop Ordering a Tray of Blood Laboratory Tests Preoperatively in Cardiac and Non-cardiac Surgery?
}

\author{
Behzad Maghsoudi* \\ Anesthesia and Intensive care Research center, Shiraz university of Medical Sciences, Iran
}

Submission: January 01, 2018; Published: February 12, 2018

*Corresponding author: Behzad Maghsoudi, Anesthesia and Intensive care Research center, Shiraz university of Medical Sciences, Shiraz, Iran,

Email: maghsodb@sums.ac.ir

Abbreviations: POE: Preoperative Evaluation; BT: Blood Tests; ROTEM: Rotational Thromboelastometry; OR: Operating Rooms

\section{Introduction}

Preoperative evaluation (POE) is an essential part of any form of surgery and anesthesia. Generally it begins with an introduction followed by history taking and physical examination. In a child or infant, most of the information's are taken from the parents. Alongside with anesthesia, POE is mandatory in surgical management. Examples include preoperative education in ambulatory laparoscopic cholecystectomy [1], risk evaluation in patients over 75 years candidates to non-cardiac and cardiac surgery [2], and hemoglobin A1c to assess preoperative diabetes risk in cardiac surgery patients [3]. Anesthetic POE includes activity level assessment such as METS classification [4], drug history, past medical and surgical history, and sensitivity to drugs and foods. Also we should not forget to allow patient speak freely about any aspect she/he consider to be important. Physical examination include airway assessment, any visible abnormalities, prominent and possible injuries, signs of upper respiratory tract infection, chest and heart auscultations, peripheral pulse assessment, assessing anxiety level along other relevant systems.

Then usually the anesthesiologist request a set of blood tests (BT), according to age, history, and type of anesthesia and surgery $[5,6]$. But the rising question is how many of these BTs are necessary preoperatively and to what extent they are really helpful? Just now there are many considerations and modifications in performing these tests as the situation in a developing country [7], before noncardiac or cardiac surgery [8] or in ambulatory surgery [9]. I think it is time for a revolution in ordering BTs before surgery. The anesthesiologists have noninvasive hemoglobin monitoring [10], along with arterial blood gas, blood sugar, electrolyte assessment, coagulation studies like rotational thromboelastometry (ROTEM), and many other laboratory tests available in seconds in both cardiac and non-cardiac operating rooms(OR). There are new monitoring devices, safer drugs, more available therapeutic options in the OR, expansion of anesthesiologists knowledge in every fields, sophisticated anesthetic drug delivery systems(inhalational and intravenous), and in a word a safer condition for anesthesia management even in currently more difficult cases.

Today many persons have a medical file by their family physician easily available by anesthesiologist. Even there are devices that can measure vital signs and send them to a doctor that are becoming popular. The market for home health monitoring devices was $\$ 10$ billion in 2010 . So what will these devices impact anesthesia practice and how will anesthesiologists should adapt their practice with them? A new IBM study using the non-invasive blood test, which analyzes blood via a wrist band and automatically sends the information to doctor could report drop in iron levels or a higher white blood cell count [11]. Cyte is another device for taking blood without a needle that can send the result to their phone, or send it to doctors [12]. If really a blood test is needed, anesthesiologists should recruit them.

By this new achievement in technology, it is time for anesthesiologists to stop many BTs like taking blood for hemoglobin in a six months old child who need herniorrhaphy. The situation is almost dramatic when a 2-5 days old neonate with transposition of great arteries is a candidate for correction. Why this neonate should be punctured for obtaining blood results, while in the operating room an arterial line is provided and all BTs could be available in minutes. I am quite sure that very soon all necessary laboratory devices will bring to the operating room, any data could be ready instantly and necessary therapeutic interventions could be done before or during operation. 


\section{References}

1. Subirana Magdaleno H, Caro Tarragó A, Olona Casas C, Díaz Padillo A, Franco Chacón M, et al. (2017) Evaluation of the impact of preoperative education in ambulatory laparoscopic cholecystectomy. A prospective, double-blind randomized trial. Cir Esp S0009-739X(17)30269-5.

2. Boccanelli A, Faggiano P (2017) Preoperative risk evaluation in patients over 75 years candidates to non-cardiac and cardiac surgery. Monaldi Arch Chest Dis 87(2): 845.

3. Gianchandani RY, Saberi S, Zrull CA, Patil PV, Jha L, et al. (2011) Evaluation of hemoglobin A1c criteria to assess preoperative diabetes risk in cardiac surgery patients. Diabetes Technol Ther 13(12):12491254 .

4. Duminda N Wijeysundera1, Rupert M Pearse2, Mark A Shulman3, Tom E F Abbott2, Elizabeth Torres4, Bernard L Croal5, et al. (2016) Measurement of Exercise Tolerance before Surgery (METS) study: a protocol for an international multicentre prospective cohort study of cardiopulmonary exercise testing prior to major non-cardiac surgery. BMJ 6(3).
5. Shannon K. Martin, Adam S Cifu (2017) Routine Preoperative Laboratory Tests for Elective Surgery. JAMA 318(6): 567-568.

6. Bock M, Fritsch G, Hepner DL (2016) Preoperative Laboratory Testing Anesthesiol Clin 34(1): 43-58.

7. Bordes J, Cungi PJ, Savoie PH, Bonnet S, Kaiser E (2015) Usefulness of routine preoperative testing in a developing country: a prospective study. Pan Afr Med J 21: 94.

8. Feely MA, Collins CS, Daniels PR, Kebede EB, Jatoi A, et al. (2013) Preoperative testing before noncardiac surgery: guidelines and recommendations. Am Fam Physician 87(6): 414-418.

9. Benarroch Gampel J, Riall TS (2013) What laboratory tests are required for ambulatory surgery? Adv Surg 47: 81-98.

10. Park YH, Lim S, Kang H1, Shin HY, Baek CW, et al. (2018) Comparison of the accuracy of noninvasive hemoglobin monitoring for preoperative evaluation between adult and pediatric patients: a retrospective study. J Clin Monit Comput.

11. Health Care Of The Future: Non-Invasive Blood Tests, Brain.

12. Yue Yuan Cyte non-invasive blood test.

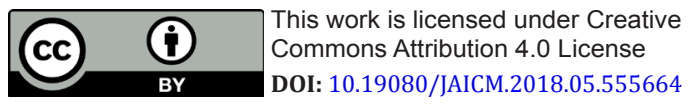

